Supporting information

High Performance Near-infrared Photodetectors Based on the Synergy Effect of Short Wavelength Light Filter and Long Wavelength Response of Perovskite/Polymer

\title{
Hybrid Structure
}

Yu Zhang ${ }^{1,2}$, Zilun Qin ${ }^{1,2}$, Xiaomin Huo ${ }^{1,2}$, Dandan Song ${ }^{1,2}$, Bo Qiao ${ }^{1,2}$, Suling Zhao ${ }^{* 1,2}$

${ }^{1}$ Key Laboratory of Luminescence and Optical Information, Beijing Jiaotong University, Ministry of Education, Beijing, 100044, China

2 Institute of Optoelectronics Technology, Beijing Jiaotong University, Beijing, 100044, China

Corresponding Author:

S.Zhao:slzhao@bjtu.edu.cn 


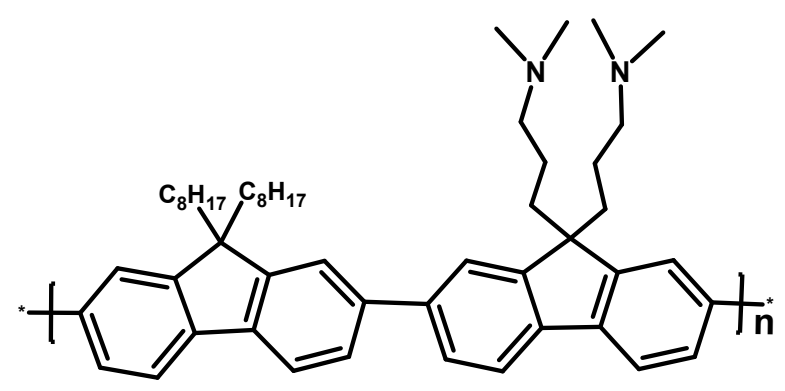

Figure S1 the molecular structure of PFN organic polymer.
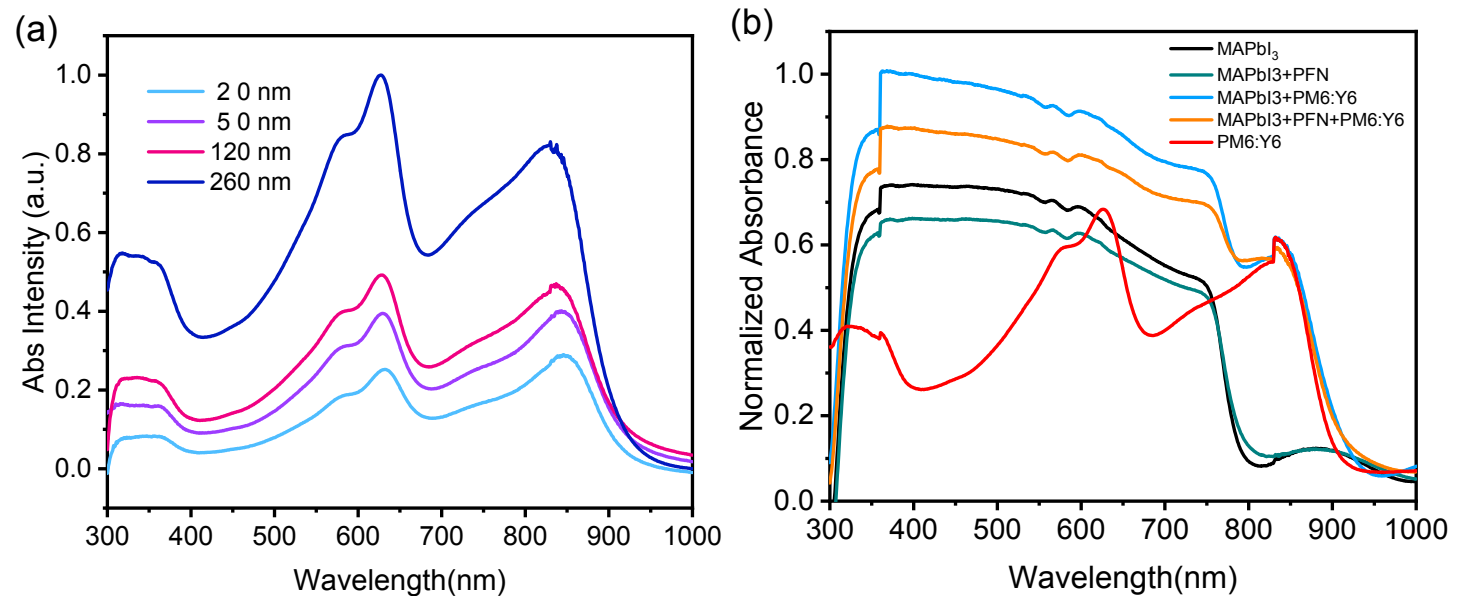

Figure S2. The absorption spectra of a) PM6:Y6 films with different thickness, b) different kinds of films with structure of $\mathrm{MAPbI}_{3} / \mathrm{MAPbI}_{3}+\mathrm{PFN} / \mathrm{MAPbI}_{3}+\mathrm{PM6:Y6/}$ $\mathrm{MAPbI}_{3}+\mathrm{PFN}+\mathrm{PM6}$ :Y6, respectively. 


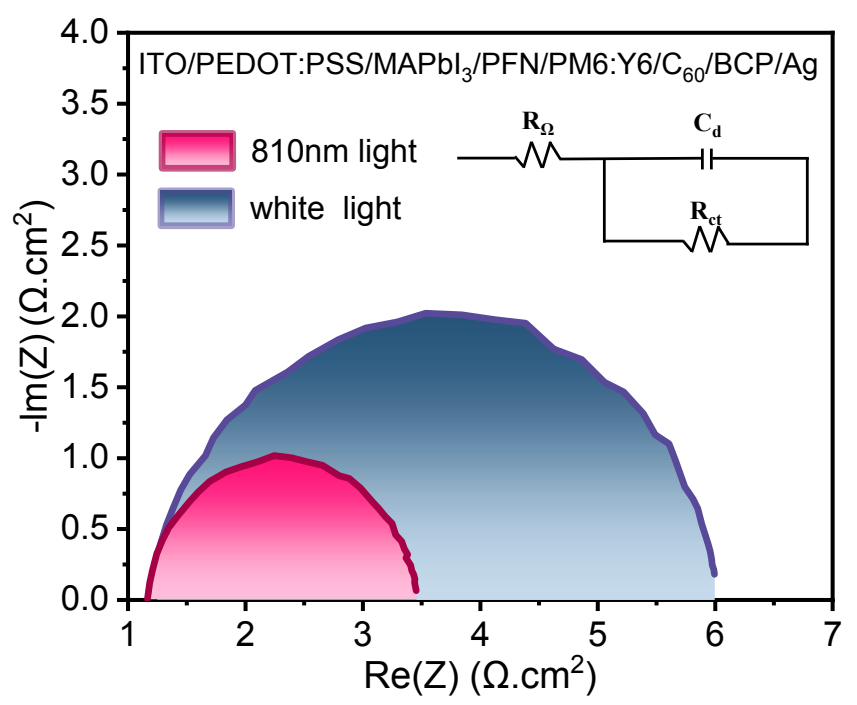

Figure S3. The electrochemical impedance spectroscopy (EIS) of SWF-NIR PD with different light

Table S1. Performs of perovskite/organic hybrid NIR photodetectors

\begin{tabular}{|c|c|c|c|c|c|c|c|c|}
\hline Structure & $\begin{array}{r}\text { Range } \\
{[\mathrm{nm}]}\end{array}$ & $\begin{array}{c}\text { Peak } \\
\mathrm{EQE} \\
{[\%]}\end{array}$ & $\begin{array}{c}\mathrm{R} \\
{\left[\mathrm{AW}^{-1}\right]}\end{array}$ & $\begin{array}{r}\text { FWHM } \\
{[\mathrm{nm}]}\end{array}$ & $\begin{array}{c}\mathrm{D}^{*} \\
\text { [Jones] }\end{array}$ & $\begin{array}{c}\text { Rise/ fall } \\
\text { time } \\
{[\mu \mathrm{s}]}\end{array}$ & $\begin{array}{l}\text { LDR } \\
{[\mathrm{dB}]}\end{array}$ & Ref. \\
\hline $\begin{array}{c}\text { Perovskite/Glass/I } \\
\mathrm{TO} / \mathrm{CuPc} / \mathrm{C}_{60}\end{array}$ & $\begin{array}{c}780- \\
900\end{array}$ & 24.8 & 0.16 & 60 & $2.83 \times 10^{11}$ & $14.4 / 32$ & $\mathrm{~N} / \mathrm{A}$ & [33] \\
\hline $\begin{array}{c}\text { MAPbI }_{3} / \text { PTB7- } \\
\text { Th: PCBM }\end{array}$ & $\begin{array}{l}750- \\
950 \\
\end{array}$ & 0.04 & 0.076 & $<50$ & - & - & 88 & [32] \\
\hline $\begin{array}{c}\mathrm{MAPbI}_{3} / \mathrm{CuSCN} / \\
\text { PM6: Y6 }\end{array}$ & $\begin{array}{l}750- \\
950 \\
\end{array}$ & 74.26 & 0.48 & $<100$ & $5.7 \times 10^{12}$ & $39.5 / 60.8$ & 162 & [34] \\
\hline $\begin{array}{c}\mathrm{MAPbI}_{3} / \mathrm{PFN} / \mathrm{PM} \\
6: \mathrm{Y} 6\end{array}$ & $\begin{array}{l}750- \\
950\end{array}$ & 83.7 & 0.577 & 131 & $1.52 \times 10^{13}$ & $1.73 / 0.98$ & 141 & $\begin{array}{l}\text { Our } \\
\text { work }\end{array}$ \\
\hline
\end{tabular}

